\title{
Refined finite element for piezoelectric laminated composite beams
}

\author{
M Ganapathi ${ }^{1}$, B P Patel ${ }^{1,3}$ and M Touratier ${ }^{2}$ \\ ${ }^{1}$ Institute of Armament Technology, Girinagar, Pune-411 025, India \\ ${ }^{2}$ LMSP, URA CNRS, ENSAM, 151, Boulevard de l'Hopital, 75013 Paris, France \\ E-mail: badripatel@hotmail.com
}

\begin{abstract}
Here, a $\mathrm{C}^{1}$ finite element is formulated for analyzing the bending/torsional behavior of rectangular piezoelectric laminated composite beams. The formulation includes transverse shear, warping due to torsion and electro-elastic coupling effects. The shear strain is represented by a cosine function of a higher order in nature, thus avoiding shear correction factors. The warping function obtained from a three-dimensional elasticity solution is incorporated in the present model. The distribution of the electric potential through the thickness is accounted for in the formulation based on the observation of the three-dimensional solution. The efficacy of the laminated piezoelectric composite element is tested comparing the results with those of analytical and three-dimensional finite element models.
\end{abstract}

\section{Introduction}

There has been increasing interest in recent years in the development of lightweight smart structures for many engineering applications. For weight optimization, structures made of composite materials having high stiffness-to-weight and strength-to-weight ratios, and sandwiches separating the stiff facings with a thick core of low density as loadbearing substrates, are preferred in aerospace engineering. Such structures are integrated with distributed piezoelectric materials that act as sensors and actuators because of the direct and converse piezoelectric effects, respectively. These structural members, being integrated with suitable control strategies and circuits, have self-monitoring and selfcontrolling capabilities. For the effective utilization of such structures, there is a growing appreciation among researchers for accurately modeling and simulating the characteristics of smart composite structures.

Studies on the behavior of smart structures have received considerable attention in the literature and reviewed by Tang 3 Address for correspondence: Mechanical Engineering Faculty, Institute of
Armament Technology, Girinagar, Pune-411 025, India. et al [1], Saravanos and Heyliger [2], Sunar and Rao [3], and Benjeddou [4]. It is shown that the structures, in general, are characterized using classical/first-order shear deformation theory coupled with either neglecting the electroelastic interactions or introducing constant electric field intensity over the piezoelectric layers with the substrate. It can be further concluded that most of the studies are devoted to the flexural analysis of laminated smart composite structures. Furthermore, a few studies have been devoted to the analysis of structures with higher-order/layer-wise theory, and also assuming the through thickness variation for the electric field of piezoelectric layers [5-10]. It is also observed that the available work on smart structures largely pertains to laminated plates. Attempts are also made in deriving the exact/analytical solutions for simple cases of geometry and boundary conditions [11-15]. As the exact solution is not possible/feasible for more general cases of loading and complicated boundary conditions, improved approximate techniques such as the finite element method have been explored for the simulation of the behavior of smart structures [5, 8, 16-18]. However, in analyzing thick smart structures, 3D finite elements [19, 20] are used which are computationally expensive. But, it is highly desirable for 


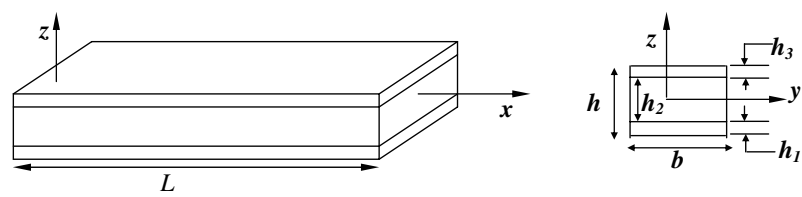

Figure 1. Laminated composite beam and coordinate system.

the designers/analysts to have a model that can capture the important 3D effects due to the through-thickness variations of displacements and stresses in thick laminates but maintain the efficiency and convenience of a 1D model. To the best of the authors' knowledge, however, the study on the torsion of laminated piezoelectric composite beam structures has not received adequate attention in the literature [21-24].

Here, a finite element for the analysis of a laminated smart beam with rectangular cross-section, having only independent generalized displacements and electric potential, is suggested by extending the recent work of Ganapathi et al [24]. The element developed is a one-dimensional model from the point of view of structural behavior and utilizes a $\mathrm{C}^{1}$ continuous function for the transverse displacement associated with bending in accordance with the refined shear deformation theory, and the torsional warping of the beam is accounted for based on a 3D elasticity solution. The electric field variation is taken as a three-dimensional one in which a quadratic form of the through-thickness variation in the piezoelectric layer is considered. The element has good features for all the standard requirements such as free from locking, spurious rigid modes, etc. The formulation includes electro-elastic coupling effects, and has no requirement of introducing an arbitrary shear correction factor as the shear strain is defined through the cosine function of a higher order nature. The efficacy of the present formulation is tested comparing the solutions with those of three-dimensional analysis. A detailed study is carried out to bring out the effect of the length-to-thickness ratio on the variation of displacements, stresses and electric potential fields due to both bending and torsional loads.

\section{Formulation}

A laminated composite beam is considered with the coordinates $x$ along the length, $y$ along the width and $z$ along the thickness directions as shown in figure 1 . The displacements in the $k$ th layer $u^{k}, v^{k}$ and $w^{k}$ at point $(x$, $y, z)$ from the median surface are expressed as functions of mid-plane displacements $u, v, w$, independent shear bending rotations $\theta_{x}$ and $\theta_{y}$ of the normal in $x z$ and $y z$ planes. They are also the functions of torsional rotation $\theta$ and independent parameter $\gamma$ for the torsional rotation gradient in the length direction as

$$
\begin{aligned}
& u^{k}(x, y, z)=u(x)-y v_{, x}(x)+f_{2}(y)\left[v_{, x}(x)+\theta_{y}(x)\right] \\
& \quad-z w_{, x}(x)+\left[f_{3}(z)+g^{k}(z)\right]\left[w_{, x}(x)+\theta_{x}(x)\right] \\
& \quad+\psi^{k}(y, z) \gamma(x) \\
& v^{k}(x, y, z)=v(x)-z \theta(x) \\
& w^{k}(x, y, z)=w(x)+y \theta(x)
\end{aligned}
$$
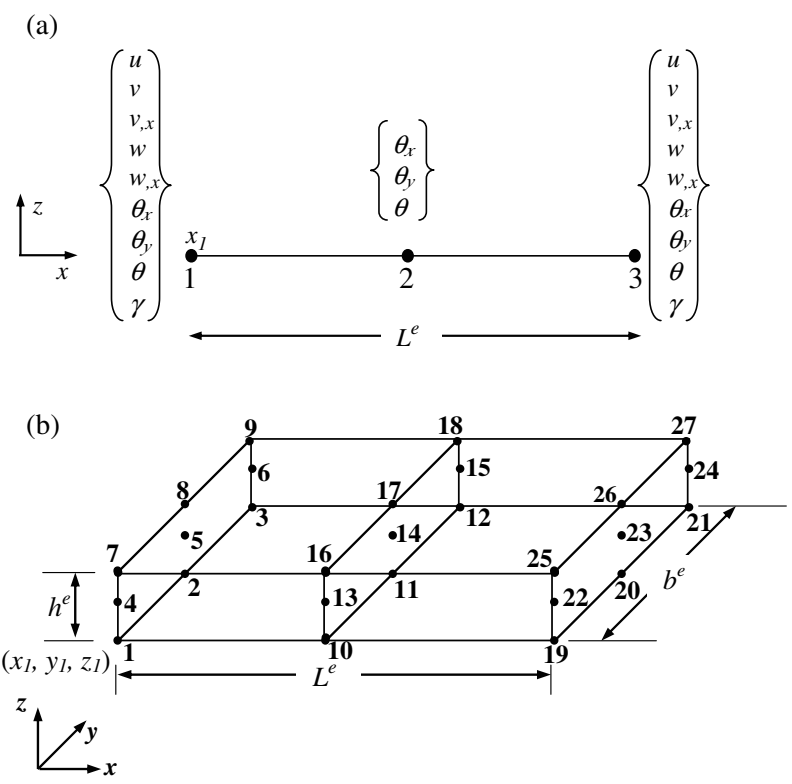

Figure 2. (a) Detail of the structural degrees of freedom of a piezoelectric beam element. (b) Description of the electrical potential degrees of freedom (1 DOF per node) in a piezoelectric beam element.

where the subscript comma denotes the partial derivative with respect to spatial coordinate succeeding it. The functions $f_{2}(y), f_{3}(z)$ and $g^{k}(z)$ are defined as

$$
\begin{gathered}
f_{2}(y)=b / \pi \sin (\pi y / b) \\
f_{3}(z)=h / \pi \sin (\pi z / h)-h / \pi b_{55} \cos (\pi z / h) \\
g^{k}(z)=a^{k} z+b^{k}
\end{gathered}
$$

where $b$ and $h$ are the width and total thickness of the beam, respectively.

In equation (2), coefficients $b^{k}$ are determined such that the contribution to the displacement component $u^{k}$, due to bending in the $x z$ plane, is continuous at the interface of adjacent layers and is zero at the mid-point of the cross-section. Finally, coefficients $b_{55}$ and $a^{k}$ in equation (2) are computed from the requirement that the transverse shear stress due to bending in the $x z$ plane is continuous at the interface of the adjacent layers and vanishes at the top and bottom surfaces of the beam. The detailed derivation of these constants $b_{55}, a^{k}$ and $b^{k}$ can be obtained from the work of Beakou and Touratier [25]. The kinematics shown in equation (1), in particular for torsion, allows one to represent the constrained torsion where axial stress is not zero, for instance near the clamped support, and free torsion, i.e. Saint-Venant torsion when $\gamma$ approaches $\theta_{, x}$, which may be realized far away from the support of a thin beam.

The torsional warping function $\psi^{k}$ used in defining the kinematics in equation (1) is the solution derived from three-dimensional elasticity equations in conjunction with the Saint-Venant assumption of torsion for a composite beam of rectangular cross section made of different layers. The general expression for $\psi^{k}$ is taken in the form of a harmonic function and is expressed as

$$
\psi^{k}=\sum_{N=1,3, \ldots}^{\infty}\left(C_{N}^{k} \sinh (\alpha z)+D_{N}^{k} \cosh (\alpha z)\right) \sin (\alpha y)+y z
$$

where $\alpha$ is defined as $N \pi / b$. 
(a)
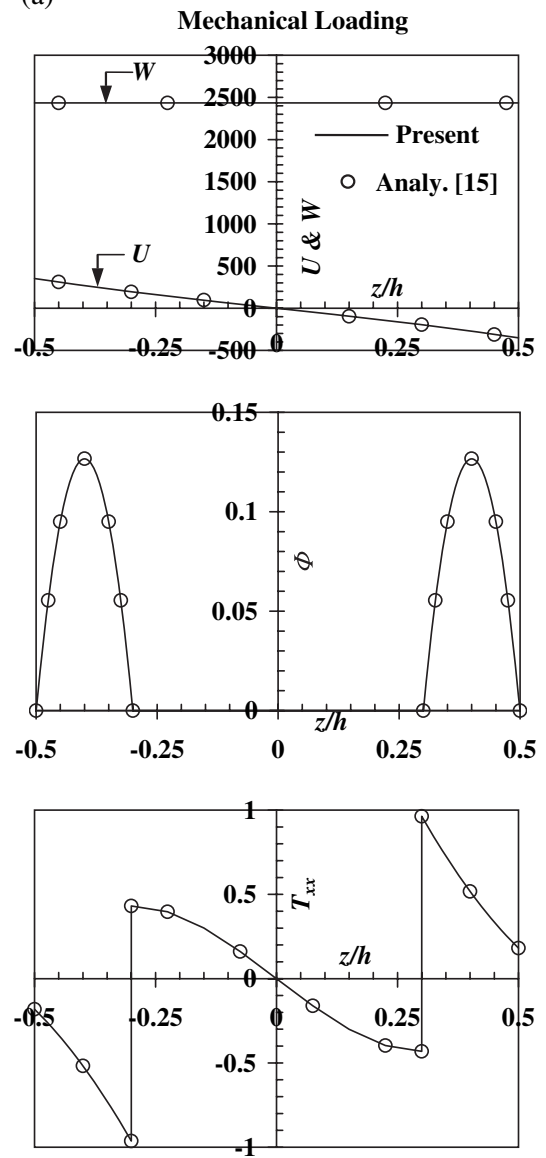
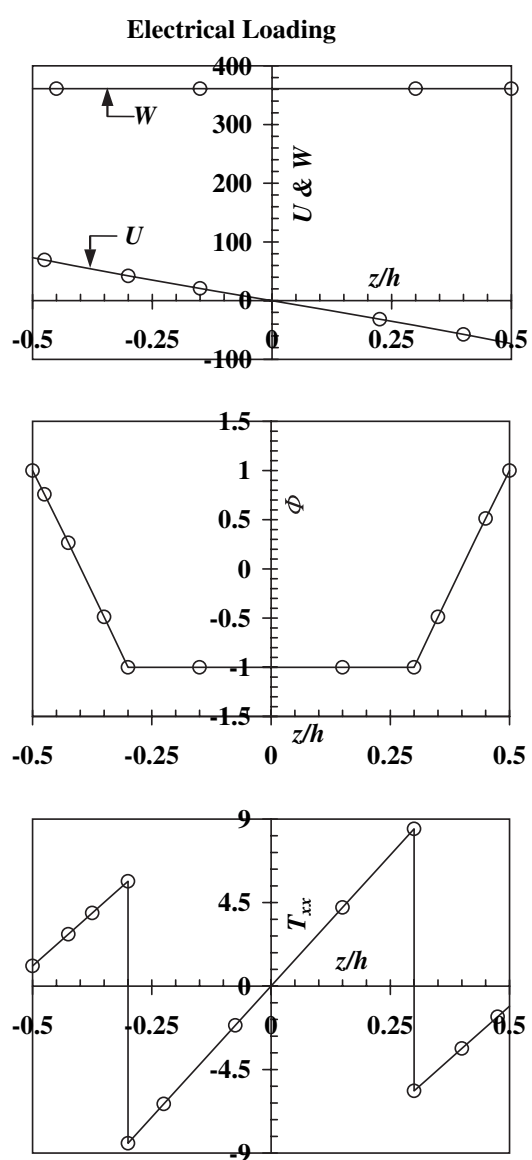

Figure 3. Comparison of nondimensional displacements $\left(U\right.$ and $W$ ), electrical potential $(\Phi)$ and stress $T_{x x}$ for a laminated piezoelectric beam subjected to mechanical (left side) and electrical (right side) loadings (a) in problem no. $1 ; L / h=10$. (b) in problem no. 1 ; $L / h=50$.

The coefficients $C_{N}^{k}$ and $D_{N}^{k}$, in equation (3), while defining the warping function for the rectangular cross section are determined such that the contribution to the displacement component $u^{k}$ due to torsion is continuous at the interface of adjacent layers, and the transverse shear stress associated with torsion is continuous at the interface of the adjacent layers and vanishes at the top and bottom surfaces of the beam [26].

The strains in terms of mid-plane deformation for the $k$ th layer can be written as

$$
\left\{\varepsilon^{k}\right\}=\left\{\begin{array}{c}
\varepsilon_{p} \\
0 \\
0
\end{array}\right\}+\left\{\begin{array}{c}
\varepsilon_{x x}^{k} \\
2 \varepsilon_{x z}^{k} \\
2 \varepsilon_{x y}^{k}
\end{array}\right\}^{\mathrm{b}}+\left\{\begin{array}{c}
\varepsilon_{x x}^{k} \\
2 \varepsilon_{x z}^{k} \\
2 \varepsilon_{x y}^{k}
\end{array}\right\}^{\mathrm{t}}
$$

where superscripts $\mathrm{b}$ and $\mathrm{t}$ denote the strain contributions due to bending and torsion, respectively.

The mid-plane strains, $\varepsilon_{p}$, the strain terms associated with bending and torsion in equation (4), are written as

$$
\begin{gathered}
\varepsilon_{p}=u_{, x} \\
\left\{\begin{array}{c}
\varepsilon_{x x}^{k} \\
2 \varepsilon_{x z}^{k} \\
2 \varepsilon_{x y}^{k}
\end{array}\right\}=\left\{\begin{array}{c}
A \\
\left(f_{3, z}+g_{, z}^{k}\right)\left(w_{, x}+\theta_{x}\right) \\
f_{2, y}\left(v_{, x}+\theta_{y}\right)
\end{array}\right\}
\end{gathered}
$$

$$
\left\{\begin{array}{c}
\varepsilon_{x x}^{k} \\
2 \varepsilon_{x z}^{k} \\
2 \varepsilon_{x y}^{k}
\end{array}\right\}^{\mathrm{t}}=\left\{\begin{array}{c}
\psi^{k} \gamma_{, x} \\
\psi_{, z}^{k} \gamma+y \theta_{, x} \\
\psi_{, y}^{k} \gamma-z \theta_{, x}
\end{array}\right\} .
$$

where $A$ in equation $(5 b)=-z w_{, x x}+\left[f_{3}(z)+g^{k}(z)\right]\left[w_{, x x}+\right.$ $\left.\theta_{x, x}\right]-y v_{, x x}+f_{2}(y)\left(v_{, x x}+\theta_{y, x}\right)$.

The total strain can be rewritten as

$$
\left\{\varepsilon^{k}\right\}=\left\{\begin{array}{c}
\varepsilon_{x x} \\
2 \varepsilon_{x z} \\
2 \varepsilon_{x y}
\end{array}\right\}=[Z]\{\bar{\varepsilon}\}
$$

where

$$
\begin{gathered}
{[Z]=\left[\begin{array}{ccccccc}
1 & y & f_{2} & z & f_{3}+g^{k} & 0 & \\
0 & 0 & 0 & 0 & 0 & 0 & \\
0 & 0 & 0 & 0 & 0 & f_{2, y} \\
& 0 & 0 & 0 & \psi^{k} \\
f_{3, z}+g_{, z}^{k} & y & \psi_{, z}^{k} & 0 \\
\{\bar{\varepsilon}\}=\left\{\begin{array}{cccccc}
u_{, x} & -v_{, x x} & v_{, x x}+\theta_{y, x} & -w_{, x x} & w_{, x x}+\theta_{x, x} \\
v_{, x}+\theta_{y} & w_{, x}+\theta_{x} & \theta_{, x} & \gamma & \gamma_{, x}
\end{array}\right\}^{\mathrm{T}} .
\end{array}\right.}
\end{gathered}
$$

For a composite laminated beam of thickness $h_{k}(k=$ $1,2,3, \ldots)$, and ply-angle $\beta_{k}(k=1,2,3, \ldots)$, the necessary expressions for computing the stiffness coefficients available in the literature [27] are used. The stress-strain relation, 

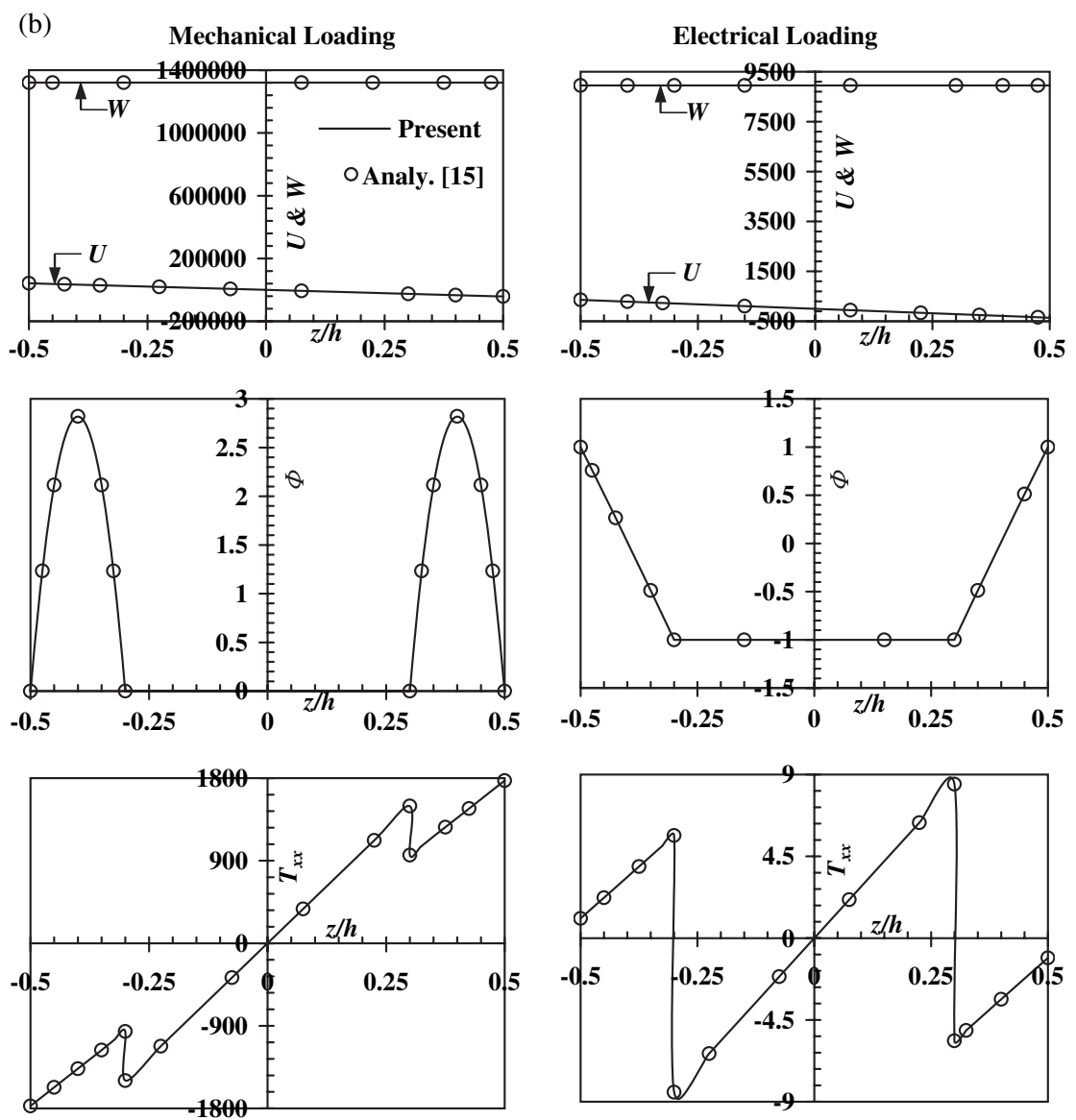

Figure 3. (Continued.)

incorporating the piezoelectric effect, for the $k$ th layer is written as

$$
\begin{gathered}
\left\{\sigma^{k}\right\}=\left[\begin{array}{ccc}
Q_{11}^{k} & 0 & Q_{16}^{k} \\
0 & Q_{44}^{k} & 0 \\
Q_{16}^{k} & 0 & Q_{66}^{k}
\end{array}\right]\left\{\varepsilon^{k}\right\} \\
-\left[\begin{array}{ccc}
0 & 0 & e_{31}^{k} \\
e_{14}^{k} & e_{24}^{k} & 0 \\
0 & 0 & e_{36}^{k}
\end{array}\right]\left\{\begin{array}{l}
E_{x}^{k} \\
E_{y}^{k} \\
E_{z}^{k}
\end{array}\right\} \\
\left\{\begin{array}{c}
D_{x}^{k} \\
D_{y}^{k} \\
D_{z}^{k}
\end{array}\right\}=\left[\begin{array}{ccc}
0 & e_{14}^{k} & 0 \\
0 & e_{24}^{k} & 0 \\
e_{31}^{k} & 0 & e_{36}^{k}
\end{array}\right]\left\{\varepsilon^{k}\right\} \\
+\left[\begin{array}{ccc}
\kappa_{11}^{k} & \kappa_{12}^{k} & 0 \\
\kappa_{21}^{k} & \kappa_{22}^{k} & 0 \\
0 & 0 & \kappa_{33}^{k}
\end{array}\right]\left\{\begin{array}{l}
E_{x}^{k} \\
E_{y}^{k} \\
E_{z}^{k}
\end{array}\right\}
\end{gathered}
$$

where $Q_{i j}^{k}(i, j=1,4,6)$ are the reduced stiffness coefficients of the $k$ th layer, $e_{i j}^{k}$ are piezoelectric coefficients, $\kappa_{i j}^{k}$ are dielectric coefficients, $D_{x}^{k}, D_{y}^{k}$ and $D_{z}^{k}$ are electrical displacements.

The electrical field intensities $E_{x}^{k}, E_{y}^{k}$ and $E_{z}^{k}$ can be related to the electric potential $\phi^{k}$ as

$$
\left\{\begin{array}{lll}
E_{x}^{k} & E_{y}^{k} & E_{z}^{k}
\end{array}\right\}^{\mathrm{T}}=-\left\{\frac{\partial \phi^{k}}{\partial x} \frac{\partial \phi^{k}}{\partial y} \frac{\partial \phi^{k}}{\partial z}\right\}^{\mathrm{T}} .
$$

The total potential energy functional $U$ of the system is given as

$$
\begin{aligned}
U(\delta) & =(1 / 2) \int_{0}^{L} \int_{-b / 2}^{b / 2} \sum_{k} \int_{h_{k}}^{h_{k+1}}\left[\left\{\sigma^{k}\right\}^{\mathrm{T}}\left\{\varepsilon^{k}\right\}\right. \\
& \left.-\left\{D^{k}\right\}^{\mathrm{T}}\left\{E^{k}\right\}\right] \mathrm{d} x \mathrm{~d} y \mathrm{~d} z \\
& -\int_{0}^{L}\{d\}^{\mathrm{T}}\left\{\begin{array}{lllllll}
f_{x} & f_{y} & f_{z} & m_{y} & m_{z} & \left.m_{x}\right\}^{\mathrm{T}} \mathrm{d} x
\end{array}\right.
\end{aligned}
$$

where $\delta$ and $L$ are the vector of the generalized coordinates and length of the beam, respectively. $f_{x}, f_{y}, f_{z}$ are the distributed forces in $x, y$ and $z$ directions and $m_{x}, m_{y}, m_{z}$ are the distributed moments about $x, y$ and $z$ axes. The vector $\{d\}$ is the vector of generalized displacements and is given by $\{d\}^{\mathrm{T}}=\left\{\begin{array}{llllll}u & v & w & \theta_{x} & \theta_{y} & \theta\end{array}\right\}$.

Using equations (6) and (7), equation (9) can be rewritten as

$$
\begin{aligned}
U(\delta) & =1 / 2 \int_{0}^{L} \int_{-b / 2}^{b / 2} \sum_{k} \int_{h_{k}}^{h_{k+1}}\left[\{\bar{\varepsilon}\}^{\mathrm{T}}[Z]^{\mathrm{T}}\left[Q^{k}\right][Z]\{\bar{\varepsilon}\}\right. \\
& -\left\{E^{k}\right\}^{\mathrm{T}}[e][Z]\{\bar{\varepsilon}\}-\{\bar{\varepsilon}\}^{\mathrm{T}}[Z]^{\mathrm{T}}[e]\left\{E^{k}\right\} \\
& \left.-\left\{E^{k}\right\}^{\mathrm{T}}[\kappa]\left\{E^{k}\right\}\right] \mathrm{d} x \mathrm{~d} y \mathrm{~d} z \\
& -\int_{0}^{L}\{d\}^{\mathrm{T}}\left\{f_{x} \quad f_{y} \quad f_{z} \quad m_{y} \quad m_{z} \quad m_{x}\right\}^{\mathrm{T}} \mathrm{d} x .
\end{aligned}
$$

The governing equations obtained by minimization of total potential energy functional given by equation (10) can be analytically/numerically solved. 
Here, the finite element approach, developing an element describing the structural field variables through three nodes along the length and the electrical field variable threedimensionally through 27 nodes, is employed. The structural fields are based on Hermite cubic functions for transverse displacements ( $v$ and $w$ according to the $\mathrm{C}^{1}$ continuity requirements), quadratic functions for rotations $\left(\theta_{x}, \theta_{y}\right.$ and $\theta$ ), linear functions for in-plane displacement, $u$, and rotation gradient pertaining to torsion, $\gamma$. The electric potential $\phi^{k}$ is interpolated using a Lagrangian function with quadratic variation along length $(x)$, width $(y)$ and thickness $(z)$ directions. Accordingly, the element needs nine structural $\left(u, v, v_{, x}, w, w_{, x}, \theta_{x}, \theta_{y}, \theta\right.$ and $\left.\gamma\right)$ degrees of freedom at both ends of the three-noded beam element and the center node requires three structural degrees of freedom $\theta_{x}, \theta_{y}$ and $\theta$, as shown in figure 2(a). Similarly, the description of the electrical potential field is based on one degree of freedom per node in all the 27 nodes of the element, i.e. 3 nodes in all directions as shown in figure 2(b). To account for the accurate variation of electrical field, each layer can be divided into many such elements through thickness along the length and width directions of beam structure. The actual element for the analysis is the integration of figures 2(a) and (b).

For obtaining the element level governing equations, the vectors $\{\bar{\varepsilon}\}$ and $\{d\}$ involved in equation (10) are expressed in terms of shape/interpolation functions, their derivatives and the vector of element level degrees of freedom/generalized displacements $\left\{\delta_{u}^{e}\right\}$ as

$$
\begin{aligned}
\{\bar{\varepsilon}\}_{10 \times 1} & =\left[B_{u}\right]_{10 \times 21}\left\{\delta_{u}^{e}\right\}_{21 \times 1} \\
\{d\}_{6 \times 1} & =\left[H_{u}\right]_{6 \times 21}\left\{\delta_{u}^{e}\right\}_{21 \times 1} .
\end{aligned}
$$

The matrices $\left[B_{u}\right],\left[H_{u}\right]$ and vector $\left\{\delta_{u}^{e}\right\}$ involved in equation (11) are defined in appendix A.

Similarly, the electric field intensity vector in an element within the layer can be expressed in terms of Lagrangian shape functions, their derivatives and element level potential degrees of freedom as

$$
\left\{E^{k}\right\}_{3 \times 1}=-\left[\bar{Z}_{\phi}\right]_{3 \times 18}\left[B_{\phi}\right]_{18 \times 27}\left\{\delta_{\phi}^{e}\right\}_{27 \times 1} .
$$

The matrices $\left[\bar{Z}_{\phi}\right],\left[B_{\phi}\right]$ and vector $\left\{\delta_{\phi}^{e}\right\}$ involved in equation (12) are given in appendix B.

Using equations (11) and (12), the total potential energy expression for the element can be written as

$U\left(\delta_{u}^{e}, \delta_{\phi}^{e}\right)=\frac{1}{2}\left\{\begin{array}{c}\delta_{u}^{e} \\ \delta_{\phi}^{e}\end{array}\right\}^{\mathrm{T}}\left[\begin{array}{ll}{\left[K_{u u}^{e}\right]} & {\left[K_{u \phi}^{e}\right]} \\ {\left[K_{\phi u}^{e}\right]} & {\left[K_{\phi \phi}^{e}\right]}\end{array}\right]\left\{\begin{array}{c}\delta_{u}^{e} \\ \delta_{\phi}^{e}\end{array}\right\}-\left\{\delta_{u}^{e}\right\}^{\mathrm{T}}\left\{F_{u}^{e}\right\}$

where the elemental stiffness matrices $\left[K_{u u}^{e}\right],\left[K_{\phi \phi}^{e}\right],\left[K_{u \phi}^{e}\right]$ and $\left[K_{\phi u}^{e}\right]$ resulting from mechanical, electrical fields and due to electro-mechanical couplings are defined as

$$
\begin{aligned}
{\left[K_{u u}^{e}\right] } & =\int_{x_{1}}^{\left(x_{1}+L^{e}\right)}\left[B_{u}\right]^{\mathrm{T}} \\
\times & {\left[\int_{y_{1}}^{\left(y_{1}+b^{e}\right)} \int_{z_{1}}^{\left(z_{1}+h^{e}\right)}[Z]^{\mathrm{T}}\left[Q^{k}\right][Z]^{\mathrm{T}} \mathrm{d} y \mathrm{~d} z\right]\left[B_{u}\right] \mathrm{d} x } \\
{\left[K_{\phi \phi}^{e}\right] } & =\int_{x_{1}}^{\left(x_{1}+L^{e}\right)}\left[B_{\phi}\right]^{\mathrm{T}} \\
& \times\left[\int_{y_{1}}^{\left(y_{1}+b^{e}\right)} \int_{z_{1}}^{\left(z_{1}+h^{e}\right)}\left[\bar{Z}_{\phi}\right]^{\mathrm{T}}[\kappa]\left[\bar{Z}_{\phi}\right]^{\mathrm{T}} \mathrm{d} y \mathrm{~d} z\right]\left[B_{\phi}\right] \mathrm{d} x \\
& =\int_{x_{1}}^{x_{1}+L^{e}}\left[B_{u}\right]^{\mathrm{T}}\left[\int_{y_{1}}^{\left(y_{1}+b^{e}\right)} \int_{z_{1}}^{\left(z_{1}+h^{e}\right)}[Z]^{\mathrm{T}}[e]\left[\bar{Z}_{\phi}\right] \mathrm{d} y \mathrm{~d} z\right]\left[B_{\phi}\right] \mathrm{d} x \\
{\left[K_{\phi u}^{e}\right] } & =\int_{x_{1}}^{x_{1}+L^{e}}\left[B_{\phi}\right]^{\mathrm{T}} \\
\times & {\left[\int_{y_{1}}^{\left(y_{1}+b^{e}\right)} \int_{z_{1}}^{\left(z_{1}+h^{e}\right)}\left[\bar{Z}_{\phi}\right]^{\mathrm{T}}[e]^{\mathrm{T}}[Z] \mathrm{d} y \mathrm{~d} z\right]\left[B_{u}\right] \mathrm{d} x . }
\end{aligned}
$$

Similarly, the elemental load vector $\left\{F_{u}^{e}\right\}$ due to applied mechanical loads may be expressed as

$$
\left\{F_{u}^{e}\right\}=\int_{x_{1}}^{\left(x_{1}+L^{e}\right)}\left[H_{u}\right]^{\mathrm{T}}\left\{\begin{array}{llllll}
f_{x} & f_{y} & f_{z} & m_{y} & m_{z} & m_{x}
\end{array}\right\}^{\mathrm{T}} \mathrm{d} x .
$$

The minimization of a total potential energy functional given by equation (13) leads to the governing equation for the element as

$$
\left[\begin{array}{cc}
{\left[K_{u u}^{e}\right]} & {\left[K_{u \phi}^{e}\right]} \\
{\left[K_{\phi u}^{e}\right]} & {\left[K_{\phi \phi}^{e}\right]}
\end{array}\right]\left\{\begin{array}{c}
\left\{\delta_{u}^{e}\right\} \\
\left\{\delta_{\phi}^{e}\right\}
\end{array}\right\}=\left\{\begin{array}{c}
\left\{F_{u}^{e}\right\} \\
\{0\}
\end{array}\right\} .
$$

The coefficients of stiffness matrices involved in the governing equation (16) can be rewritten as the product of terms having thickness $(z)$ and width $(y)$ co-ordinates, and the term containing $x$. In the present study, while performing the integration, terms having thickness and width co-ordinates are explicitly integrated, whereas the terms with $x$ are evaluated based on full integration, using the three point Gauss integration rule.

Following the finite element assembly procedure, the governing equations for the beam structure are obtained as

$$
\left[\begin{array}{cc}
{\left[K_{u u}^{G}\right]} & {\left[K_{u \phi}^{G}\right]} \\
{\left[K_{\phi u}^{G}\right]} & {\left[K_{\phi \phi}^{G}\right]}
\end{array}\right]\left\{\begin{array}{c}
\left\{\delta_{u}^{G}\right\} \\
\left\{\delta_{\phi}^{G}\right\}
\end{array}\right\}=\left\{\begin{array}{c}
\left\{F_{u}^{G}\right\} \\
\{0\}
\end{array}\right\}
$$

where superscript $G$ denotes the global matrices and vector.

Equation (17) can be solved using any standard method.

\section{Results and discussion}

The aim of the present study is to examine the efficacy of this new element for analyzing the bending and torsional behaviors of the laminated or sandwich piezoelectric beam. The choice of the interpolation functions made here in developing the element allows us to have the same order of interpolation for both $w_{, x}$ and $\theta_{x}, v_{, x}$ and $\theta_{y}$ in the definition of shear 

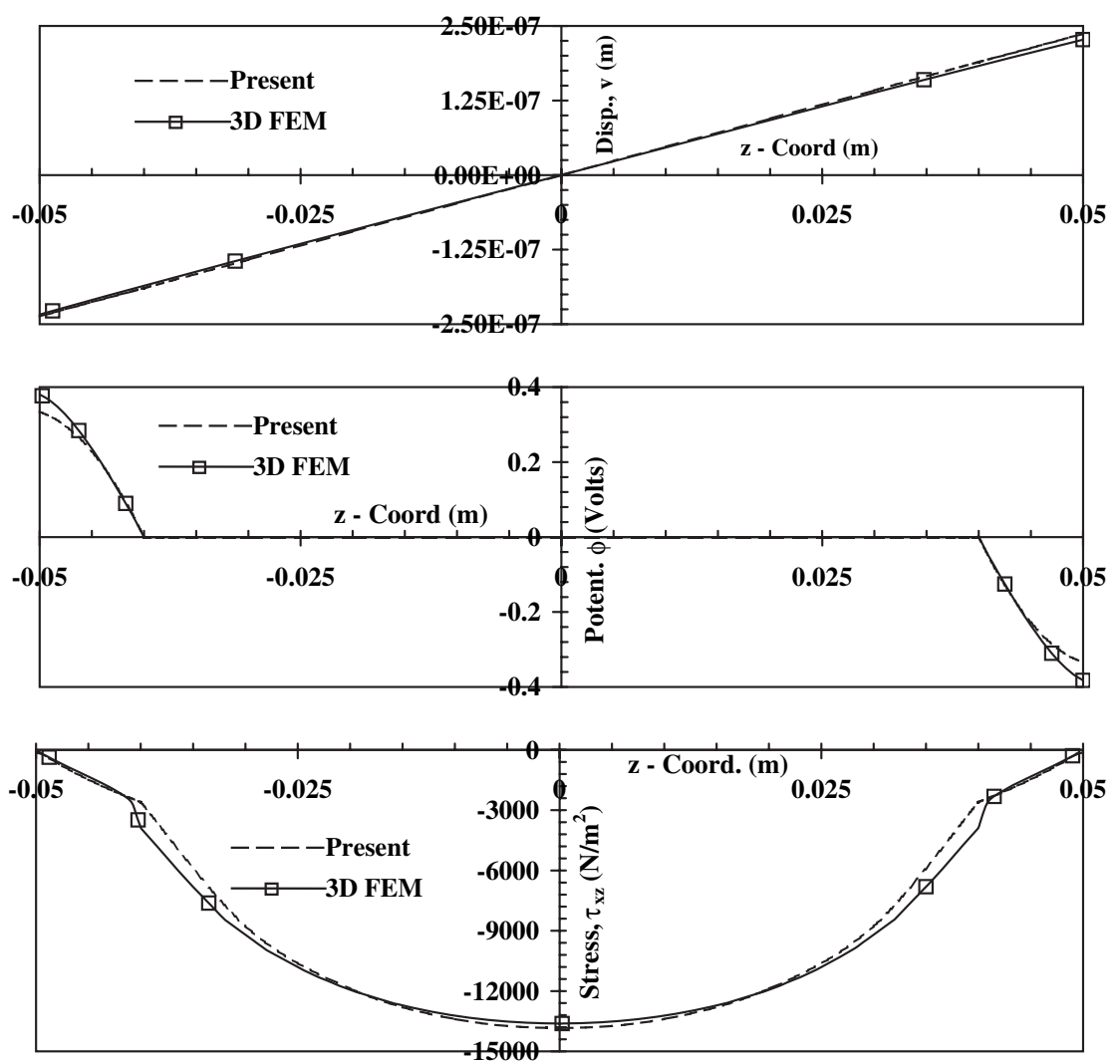

Figure 4. Distribution of displacements, electrical potential and stress along the thickness (at $(x, y)=(0.5 \mathrm{~m}, 0.03 \mathrm{~m}))$ due to $1 \mathrm{~N} \mathrm{~m}$ torque applied at the free end in problem no. 2.

strain and permits the avoidance of transverse shear locking phenomena. Similarly, $\theta_{, x}$ and $\gamma$ in the torsional strain are interpolated with the same degree polynomial which recovers the Saint-Venant torsion $\left(\gamma=\theta_{, x}\right)$. The element behaves very well for both thick and thin situations as highlighted in the work of Ganapathi et al [24]. Furthermore, it has been demonstrated [24] that this element has no spurious mode and is represented by correct rigid body modes pertaining to flexure and torsion when exact integration is applied to evaluate all the strain energy terms and, for the sake of brevity, these results are not presented here.

The problems chosen for testing the performance of the element are given below as:

\section{Flexural analysis}

Problem 1. Simply supported laminated piezoelectric composite beam subjected to mechanical/electrical loads [15]. The properties such as elastic, piezoelectric/dielectric coefficients used are:

For skin (PZT4): $\quad C_{11}=C_{22}=139 \mathrm{GPa}, C_{12}=77.8 \mathrm{GPa}$ $C_{13}=C_{23}=74.3 \mathrm{GPa}, C_{33}=115 \mathrm{GPa}, C_{44}=C_{55}=$ $25.6 \mathrm{GPa}, C_{66}=30.6 \mathrm{GPa} ; e_{31}=-5.2 \mathrm{C} \mathrm{m}^{-2}, e_{32}=$ $-5.2 \mathrm{C} \mathrm{m}^{-2}, e_{33}=15.1 \mathrm{C} \mathrm{m}^{-2}, e_{15}=e_{24}=12.7 \mathrm{C} \mathrm{m}^{-2}$; $\kappa_{11}=\kappa_{22}=13.06 \times 10^{-9} \mathrm{~F} \mathrm{~m}^{-1}, \kappa_{33}=11.51 \times 10^{-9} \mathrm{~F} \mathrm{~m}^{-1}$.

For core (graphite epoxy): $\quad C_{11}=134.86 \mathrm{GPa}, C_{12}=C_{13}=$ $5.1563 \mathrm{GPa}, C_{22}=C_{33}=14.352 \mathrm{GPa}, C_{23}=7.1329 \mathrm{GPa}$
$C_{44}=3.60955 \mathrm{GPa}, C_{55}=C_{66}=5.654 \mathrm{GPa} ; \kappa_{11}=\kappa_{22}=$ $0.031 \times 10^{-9} \mathrm{~F} \mathrm{~m}^{-1}, \kappa_{33}=0.0266 \times 10^{-9} \mathrm{~F} \mathrm{~m}^{-1}$.

Geometrical properties: Skin thickness $h_{1}=h_{3}=0.2 \mathrm{~h}$, core thickness $h_{2}=0.8 \mathrm{~h}$; width $b=0.0125 \mathrm{~m} ; L / h$ is varied as 10 and 50 .

\section{Torsional analysis}

Problem 2. Cantilever piezoelectric (PZT/epoxy) sandwich beam subjected to mechanical/electrical loads. The properties such as elastic, piezoelectric/dielectric coefficients considered here are [28]:

For face (PZT/epoxy): $\quad E_{1}=37.8 \mathrm{GPa}, E_{2}=6.98 \mathrm{GPa}$, $E_{3}=6.98 \mathrm{GPa}, G_{12}=G_{23}=G_{13}=2.89 \mathrm{GPa}$, $v_{12}=v_{23}=v_{13}=0.26 ; d_{31}=-274 \times 10^{-12} \mathrm{~m} \mathrm{~V}^{-1}$, $d_{32}=-120 \times 10^{-12} \mathrm{~m} \mathrm{~V}^{-1}, d_{33}=374 \times 10^{-12} \mathrm{~m} \mathrm{~V}^{-1}$; $\kappa_{11}=\kappa_{22}=\kappa_{33}=16.5 \times 10^{-9} \mathrm{~F} \mathrm{~m}^{-1}$.

For core: $\quad E=73 \mathrm{GPa}, G=29.2 \mathrm{GPa}, v=0.25$.

Geometrical parameters: Width $(b)=0.06 \mathrm{~m}$, total thickness of the beam $h=0.1 \mathrm{~m}, h_{1}=0.01 \mathrm{~m}, h_{2}=0.08 \mathrm{~m}$, $h_{3}=0.01 \mathrm{~m}$, length $L=1 \mathrm{~m}$.

The boundary conditions used are:

simply supported case: $u=v=w=0$ at $x=0, L$

Clamped end: $\quad u=v=v_{, x}=w=w_{, x}=\theta_{x}=\theta_{y}=\theta=$ $\gamma=0$ at $x=0$ 


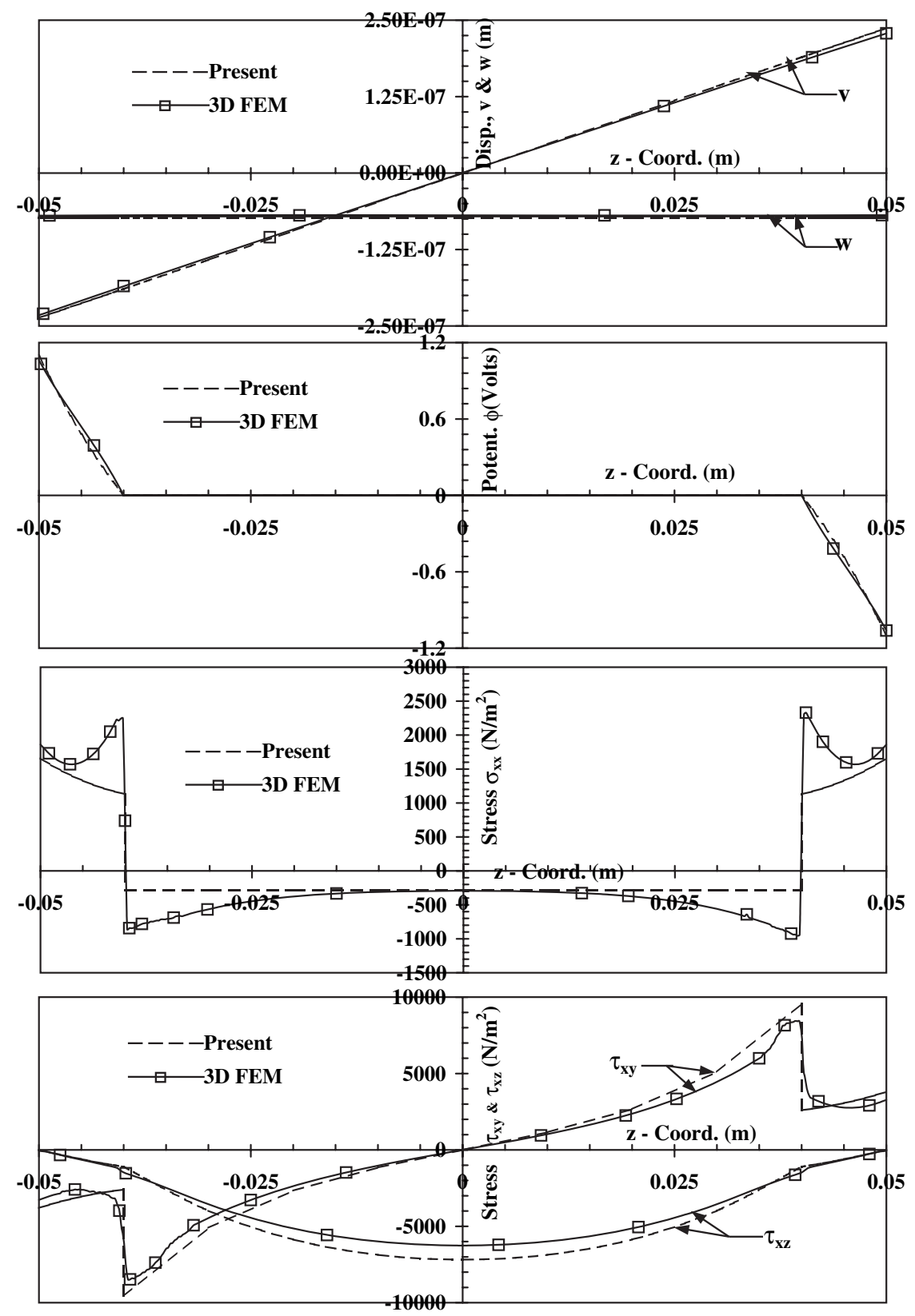

Figure 5. Distribution of displacements, electrical potential and stresses along the thickness (at $(x, y)=(0.5 \mathrm{~m}, 0.015 \mathrm{~m}))$ due to $1 \mathrm{~N} \mathrm{~m}$ torque applied at the free end in problem no. 2.

Based on progressive mesh refinement, mesh idealization of the 10-element along the length and the 1-element in each piezoelectric layer is found to be adequate to model problem 1 for the bending analysis. For the mechanical case, a uniform pressure of $0.05 \mathrm{MPa}$ is assumed on the top surface, in addition to zero electrical potential conditions at the top and bottom surfaces as well as the core of the beam. For the electrical loading situation, the potential of $100 \mathrm{~V}$ at the top and bottom surfaces of the beam, and $-100 \mathrm{~V}$ at the top and bottom surfaces of the core are applied. The normalized displacements/stress due to mechanical load $\left(U=u(0,0, z) C_{00} /(h q) ; W=w(L / 2,0, z) C_{00} /(h q) ;\right.$ $\Phi=\phi(L / 2,0, z) C_{00} /\left(h q E_{0}\right) ; T_{11}=\sigma_{11}(L / 2,0, z) / q$ where $C_{00}=134.86 \mathrm{GPa}$ and $E_{0}=1 \times 10^{10} \mathrm{~V} \mathrm{~m}^{-1}$ ) and applied electrical field $\left(U=u(0,0, z) E_{0} / V_{0} ; W=\right.$
$w(L / 2,0, z) E_{0} / V_{0} ; \quad \Phi=\phi(L / 2,0, z) / V_{0} ; \quad T_{11}=$ $\sigma_{11}(L / 2,0, z) h E_{0} /\left(C_{00} V_{0}\right)$ where $\left.V_{0}=100 \mathrm{~V}\right)$ obtained through the thickness of the simply supported piezoelectric beam using the present element are shown in figures 3(a) and (b) along with the exact analytical solutions [15] for both the thick and thin cases $(L / h=10$ and 50). The present model predicts the solutions accurately and they match very well with the available analytical results. It is further inferred from figure 3(b) that the element does not lock and is free from the locking phenomenon, irrespective of the type of loading.

Next, the torsional characteristics of the piezoelectric beam is analyzed considering a cantilever with geometrical parameters and material properties given in problem 2 for both mechanical and electrical loading situations. Unlike in problem 1, here, the skins are treated as PZT/epoxy 

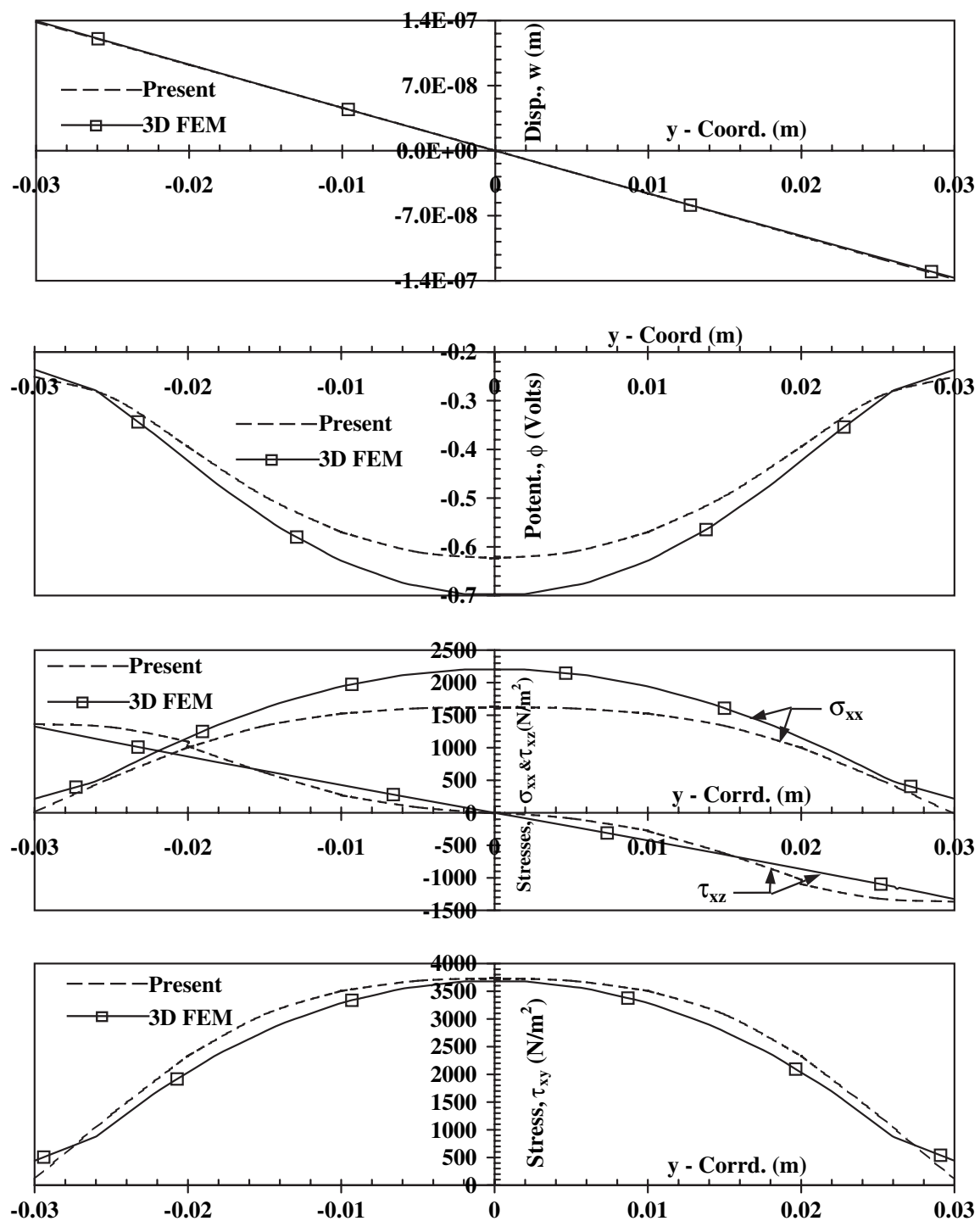

Figure 6. Distribution of displacements, electrical potential and stresses along the width (at $(x, z)=(0.5 \mathrm{~m}, 0.045 \mathrm{~m}))$ due to $1 \mathrm{~N} \mathrm{~m}$ torque applied at the free end in problem no. 2.

layers. The lamination scheme considered for this case is $-45^{\circ}$ (bottom skin) $/ 0^{\circ}$ (core) $/ 45^{\circ}$ (top skin). A torque of $1 \mathrm{~N} \mathrm{~m}$ is assumed at the free end of the cantilever beam for the mechanical case, whereas the electrical potential of $100 \mathrm{~V}$ at the bottom surface of the beam and $-100 \mathrm{~V}$ at the top surface of the beam are considered for the electrical loading case. The electrical potential conditions in the core and along the interfaces of the laminate are treated as zero values for all the analyses. A detailed convergence study is conducted by increasing the number of elements in the thickness as well as the width directions and also increasing number of terms in the warping function, and, for the sake of brevity, these results are not presented here. For the chosen problem here, the finite element solutions are evaluated based on the 14-term in the warping function in conjunction with 8-, 4- and 6-element idealization along the length, thickness and width of the each piezoelectric layers of the sandwich beam, respectively. The numerical results generated here for deflections and stresses along the thickness (at $x / L=0.5$, $y / b=0.5$ and 0.25 ) and width (at $x / L=0.5, z / h=$ $0.45)$ directions of the beam are plotted in figures 4-7 and compared with those of the three-dimensional finite element model using ANSYS 5.6. Furthermore, the present model exhibits the presence of axial stress due to the torsional load and this is due to the fact that the formulation accounts for the constrained torsion. It is revealed from these figures that the results predicted by the present element are, in general, in fairly good agreement with three-dimensional solutions. Some differences noticed in the results between the present one and the 3D model for the distributions of stress/potential fields are obvious, as the state of stress/potential distributions for this class of problems is three-dimensional in nature. It can be noted that the element treated here is essentially a three-dimensional representation of an electrical field, but is structurally a one-dimensional one. It is worthwhile giving the details of the mesh using an 8-noded brick element (with 4-DOF per node) for 3D modeling of the problem from ANSYS. The beam for the torsional analysis is discretized, 

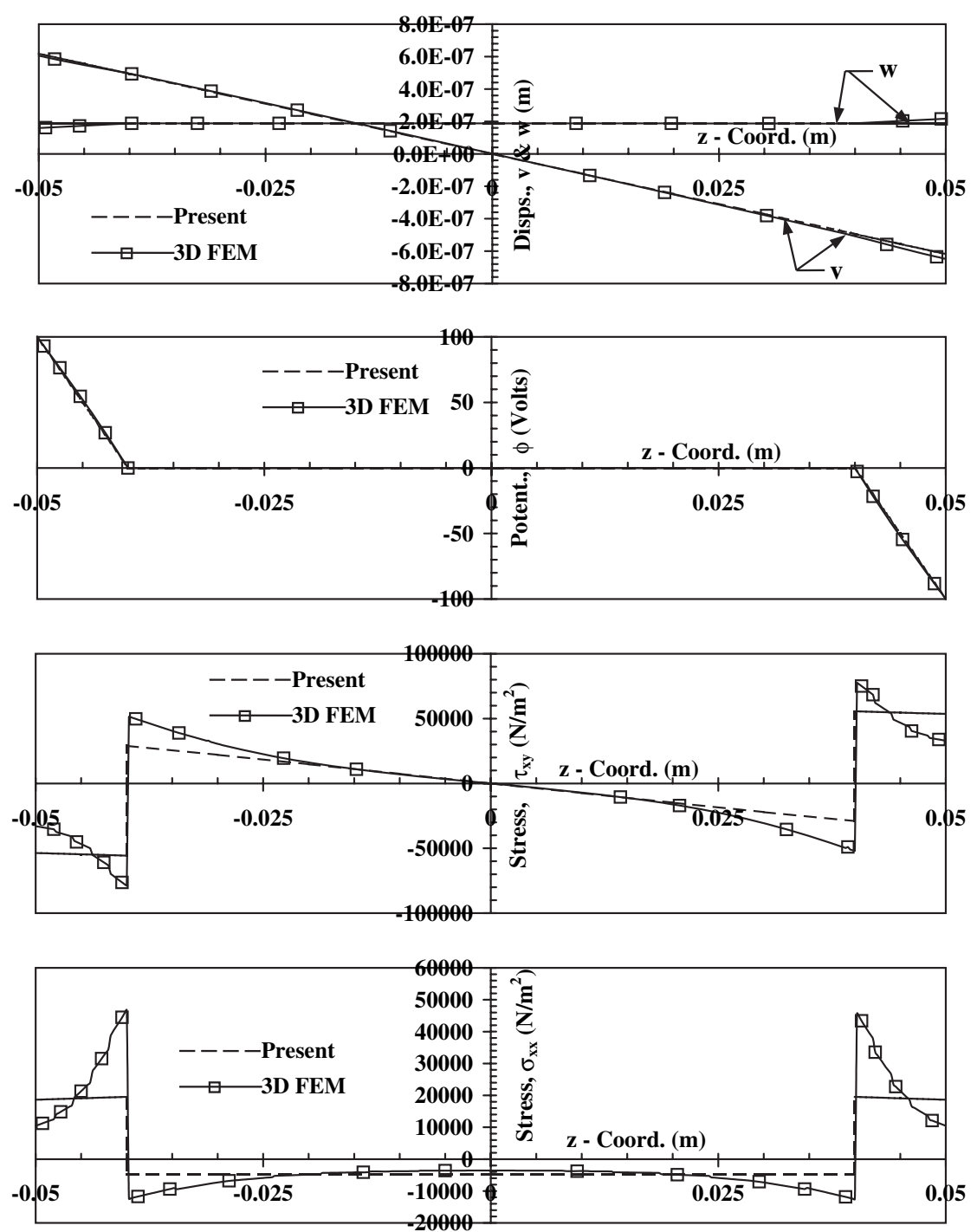

Figure 7. Distribution of displacements, electrical potential and stresses along the thickness (at $(x, y)=(0.5 \mathrm{~m}, 0.015 \mathrm{~m}))$ due to electrical load in problem no. 2 .

using $20 \times 30$ mesh along the length and width directions, 20 elements in each skin (piezoelectric layer) and 40 elements in the core along the thickness direction. It may be opined from these studies that, for the given accuracy, the present formulation leads to significant reduction in computational time and memory requirements in comparison with those of rigorous $3 \mathrm{D}$ analyses.

\section{Conclusions}

The finite element suggested for analyzing laminated/sandwich piezoelectric beams with rectangular cross section, is based on refined shear deformation theory, and the inclusion of threedimensional effects for warping and the distribution of electrical potential field. The present element, in general, performs well for both flexural and torsional behaviors of a laminated piezoelectric beam. The presence of axial stress while the beam is subjected to torsional load is brought out, as the constrained torsion is incorporated in the model. The formulation outlined here results in significant reduction in computational time and memory.

\section{Acknowledgments}

This study was supported by Indo French Centre for the Promotion of Advanced Research (Centre Franco-Indien Pour La Promotion De La Recherche Avancee), Department of Science and Technology, Government of India, through the grant of Project No. 2308-1.

\section{Appendix A}

The various matrices involved in equation (11) are

$\left[B_{u}\right]=\left[\begin{array}{cccccc}n_{1, x} & 0 & 0 & 0 & 0 & 0 \\ 0 & -\bar{h}_{1, x x} & -\bar{h}_{2, x x} & 0 & 0 & 0 \\ 0 & \bar{h}_{1, x x} & \bar{h}_{2, x x} & 0 & 0 & 0 \\ 0 & 0 & 0 & -\bar{h}_{1, x x} & -\bar{h}_{2, x x} & 0 \\ 0 & 0 & 0 & \bar{h}_{1, x x} & \bar{h}_{2, x x} & s_{1, x} \\ 0 & \bar{h}_{1, x} & \bar{h}_{2, x} & 0 & 0 & 0 \\ 0 & 0 & 0 & \bar{h}_{1, x} & \bar{h}_{2, x} & s_{1} \\ 0 & 0 & 0 & 0 & 0 & 0 \\ 0 & 0 & 0 & 0 & 0 & 0 \\ 0 & 0 & 0 & 0 & 0 & 0\end{array}\right.$




$$
\begin{aligned}
& \begin{array}{cccccccc}
0 & 0 & 0 & 0 & 0 & 0 & n_{2, x} & 0 \\
0 & 0 & 0 & 0 & 0 & 0 & 0 & -\bar{h}_{3, x x}
\end{array} \\
& \begin{array}{llllllll}
s_{1, x} & 0 & 0 & 0 & s_{2, x} & 0 & 0 & \bar{h}_{3, x x}
\end{array} \\
& \begin{array}{cccccccc}
0 & 0 & 0 & 0 & 0 & 0 & 0 & 0
\end{array} \\
& \begin{array}{rrrrrrrr}
0 & 0 & 0 & s_{2, x} & 0 & 0 & 0 & 0
\end{array} \\
& \begin{array}{cccccccc}
s_{1} & 0 & 0 & 0 & s_{2} & 0 & 0 & \bar{h}_{3, x} \\
0 & 0 & 0 & s_{2} & 0 & 0 & 0 & 0
\end{array} \\
& \begin{array}{llllllll}
0 & s_{1, x} & 0 & 0 & 0 & s_{2, x} & 0 & 0
\end{array} \\
& \begin{array}{llllllll}
0 & 0 & n_{1} & 0 & 0 & 0 & 0 & 0
\end{array} \\
& \begin{array}{llllllll}
0 & 0 & n_{1, x} & 0 & 0 & 0 & 0 & 0
\end{array} \\
& \left.\begin{array}{ccccccc}
0 & 0 & 0 & 0 & 0 & 0 & 0 \\
-\bar{h}_{4, x x} & 0 & 0 & 0 & 0 & 0 & 0 \\
\bar{h}_{4, x x} & 0 & 0 & 0 & s_{3, x} & 0 & 0 \\
0 & -\bar{h}_{3, x x} & -\bar{h}_{4, x x} & 0 & 0 & 0 & 0 \\
0 & \bar{h}_{3, x x} & \bar{h}_{4, x x} & s_{3, x} & 0 & 0 & 0 \\
\bar{h}_{4, x} & 0 & 0 & 0 & s_{3} & 0 & 0 \\
0 & \bar{h}_{3, x} & \bar{h}_{4, x} & s_{3} & 0 & 0 & 0 \\
0 & 0 & 0 & 0 & 0 & s_{3, x} & 0 \\
0 & 0 & 0 & 0 & 0 & 0 & n_{2} \\
0 & 0 & 0 & 0 & 0 & 0 & n_{2, x}
\end{array}\right] \\
& {\left[H_{u}\right]=\left[\begin{array}{ccccccccccc}
n_{1} & 0 & 0 & 0 & 0 & 0 & 0 & 0 & 0 & 0 & 0 \\
0 & \bar{h}_{1} & \bar{h}_{2} & 0 & 0 & 0 & 0 & 0 & 0 & 0 & 0 \\
0 & 0 & 0 & \bar{h}_{1} & \bar{h}_{2} & 0 & 0 & 0 & 0 & 0 & 0 \\
0 & 0 & 0 & 0 & 0 & s_{1} & 0 & 0 & 0 & s_{2} & 0 \\
0 & 0 & 0 & 0 & 0 & 0 & s_{1} & 0 & 0 & 0 & s_{2} \\
0 & 0 & 0 & 0 & 0 & 0 & 0 & s_{1} & 0 & 0 & 0
\end{array}\right.}
\end{aligned}
$$

$\left.\begin{array}{cccccccccc}0 & n_{2} & 0 & 0 & 0 & 0 & 0 & 0 & 0 & 0 \\ 0 & 0 & \bar{h}_{3} & \bar{h}_{4} & 0 & 0 & 0 & 0 & 0 & 0 \\ 0 & 0 & 0 & 0 & \bar{h}_{3} & \bar{h}_{4} & 0 & 0 & 0 & 0 \\ 0 & 0 & 0 & 0 & 0 & 0 & s_{3} & 0 & 0 & 0 \\ 0 & 0 & 0 & 0 & 0 & 0 & 0 & s_{3} & 0 & 0 \\ s_{2} & 0 & 0 & 0 & 0 & 0 & 0 & 0 & s_{3} & 0\end{array}\right]$

where $\left(n_{1}, n_{2}\right),\left(s_{1}, s_{2}, s_{3}\right)$ and $\left(\bar{h}_{1}, \bar{h}_{2}, \bar{h}_{3}, \bar{h}_{4}\right)$ are the set of shape functions (linear, quadratic and Hermite) used for interpolating the structural field variables $(u, \gamma),\left(\theta_{x}, \theta_{y}, \theta\right)$ and $(v, w)$, respectively. They are defined as:

$$
\begin{gathered}
n_{1}=1-\left(x-x_{1}\right) / L^{e} ; \quad n_{2}=\left(x-x_{1}\right) / L^{e} \\
s_{1}=1-3\left(x-x_{1}\right) / L^{e}+2\left(\frac{x-x_{1}}{L^{e}}\right)^{2} ; \\
s_{2}=4\left(x-x_{1}\right) / L^{e}-4\left(\frac{x-x_{1}}{L^{e}}\right)^{2} ; \\
s_{3}=-\left(x-x_{1}\right) / L^{e}+2\left(\frac{x-x_{1}}{L^{e}}\right)^{2} \\
\bar{h}_{1}=1-3\left(\frac{x-x_{1}}{L^{e}}\right)^{2}+2\left(\frac{x-x_{1}}{L^{e}}\right)^{3} ; \\
\bar{h}_{2}=\left(x-x_{1}\right)\left(1-\frac{x-x_{1}}{L^{e}}\right)^{2} ; \\
\bar{h}_{3}=3\left(\frac{x-x_{1}}{L^{e}}\right)^{2}-2\left(\frac{x-x_{1}}{L^{e}}\right)^{3} \\
\bar{h}_{4}=\left(x-x_{1}\right)\left[\left(\frac{x-x_{1}}{L^{e}}\right)^{2}-\frac{x-x_{1}}{L^{e}}\right] .
\end{gathered}
$$

Here, $x_{1}$ is the global $x$-coordinate of first node of an element.
The vector of elemental structural degrees of freedom is denoted as:

$$
\begin{aligned}
& \left\{\delta_{u}^{e}\right\}^{\mathrm{T}}=\left\{\begin{array}{llllllllll}
u_{1} & v_{1} & v_{, x 1} & w_{1} & w_{, x 1} & \theta_{x 1} & \theta_{y 1} & \theta_{1} & \gamma_{1} & \theta_{x 2}
\end{array}\right. \\
& \begin{array}{lllllllll}
\theta_{y 2} & \theta_{2} & u_{3} & v_{3} & v_{, x 3} & w_{3} & w_{, x 3} & \theta_{x 3} & \theta_{y 3}
\end{array} \\
& \left.\theta_{3} \gamma_{3}\right\} \text {. }
\end{aligned}
$$

\section{Appendix B}

The matrices involved while defining the electrical field intensities in equations (12) are

$$
\left[\bar{Z}_{\phi}\right]=\left[\begin{array}{ccccccccccc}
l_{1} & l_{2} & l_{3} & l_{4} & l_{5} & l_{6} & l_{7} & l_{8} & l_{9} & 0 & 0 \\
0 & 0 & 0 & 0 & 0 & 0 & 0 & 0 & 0 & l_{1, y} & l_{2, y} \\
0 & 0 & 0 & 0 & 0 & 0 & 0 & 0 & 0 & l_{1, z} & l_{2, z} \\
& 0 & 0 & 0 & 0 & 0 & 0 & 0 \\
& l_{3, y} & l_{4, y} & l_{5, y} & l_{6, y} & l_{7, y} & l_{8, y} & l_{9, y} \\
& l_{3, z} & l_{4, z} & l_{5, z} & l_{6, z} & l_{7, z} & l_{8, z} & l_{9, z}
\end{array}\right] .
$$

The non-zero elements of matrix $\left[B_{\phi}\right]$ are:

$$
\begin{array}{cc}
B_{\phi}(i, i)=s_{1, x} ; & B_{\phi}(i, i+9)=s_{2, x} ; \\
B_{\phi}(i, i+18)=s_{3, x} ; & B_{\phi}(i+9, i)=s_{1} ; \\
B_{\phi}(i+9, i+9)=s_{2} ; & B_{\phi}(i+9, i+18)=s_{3} \\
(i=1,2, \ldots, 9) .
\end{array}
$$

The shape functions $\left(l_{1}, l_{2}, \ldots, l_{9}\right)$ which are expressed as the product of quadratic Lagrangian polynomials in $y\left(n_{i}^{y}, i=\right.$ $1,2,3)$ and $z\left(n_{i}^{z}\right)$ directions, and $\left(s_{1}, s_{2}, s_{3}\right)$ quadratic shape functions along the length $(x)$ employed for the interpolation of the electrical potential field are:

$$
\begin{gathered}
l_{1}=n_{1}^{y} n_{1}^{z} ; \quad l_{2}=n_{2}^{y} n_{1}^{z} ; \quad l_{3}=n_{3}^{y} n_{1}^{z} ; \\
l_{4}=n_{1}^{y} n_{2}^{z} ; \quad l_{5}=n_{2}^{y} n_{2}^{z} ; \quad n_{3}^{z} ; \quad l_{3}=n_{2}^{y} n_{3}^{z} ; \quad n_{3}^{y} n_{3}^{z} \\
n_{1}^{y}=1-3\left(y-y_{1}\right) / b^{e}+2\left(\frac{y-y_{1}}{b^{e}}\right)^{2} ; \\
n_{2}^{y}=4\left(y-y_{1}\right) / b^{e}-4\left(\frac{y-y_{1}}{b^{e}}\right)^{2} ; \\
n_{3}^{y}=-\left(y-y_{1}\right) / b^{e}+2\left(\frac{y-y_{1}}{b^{e}}\right)^{2} \\
n_{1}^{z}=1-3\left(z-z_{1}\right) / h^{e}+2\left(\frac{z-z_{1}}{h^{e}}\right)^{2} ; \\
n_{2}^{z}=4\left(z-z_{1}\right) / h^{e}-4\left(\frac{z-z_{1}}{h^{e}}\right)^{2} ; \\
n_{3}^{z}=-\left(z-z_{1}\right) / h^{e}+2\left(\frac{z-z_{1}}{h^{e}}\right)^{2} \\
s_{3}=-\left(x-x_{1}\right) / L^{e}+2\left(\frac{x-x_{1}}{L^{e}}\right)^{2} \\
s_{2}=1-3\left(x-x_{1}\right) / L^{e}+2\left(\frac{x-x_{1}}{L^{e}}\right)^{2} ; \\
L^{e}
\end{gathered}
$$


Here, $\left(x_{1}, y_{1}, z_{1}\right)$ are the global coordinates of the first node of an element.

The vector of element level electrical potential degrees of freedom is described as:

$$
\begin{aligned}
\left\{\delta_{\phi}^{e}\right\}^{\mathrm{T}}= & \left\{\begin{array}{llllllllll}
\phi_{1} & \phi_{2} & \phi_{3} & \phi_{4} & \phi_{5} & \phi_{6} & \phi_{7} & \phi_{8} & \phi_{9} & \phi_{10} \\
& \phi_{11} & \phi_{12} & \phi_{13} & \phi_{14} & \phi_{15} & \phi_{16} & \phi_{17} & \phi_{18} & \phi_{19} \\
& \phi_{20} & \phi_{21} & \phi_{22} & \phi_{23} & \phi_{24} & \phi_{25} & \phi_{26} & \phi_{27} y
\end{array}\right\} .(\mathrm{B} .3)
\end{aligned}
$$

\section{References}

[1] Tang Y Y, Noor A K and Xu K 1996 Assessment of computational models for thermoelectroelastic multilayered plates Comput. Struct. 61 915-33

[2] Saravanos D A and Heyliger P R 1999 Mechanics and computational models for laminated piezoelectric beams, plates, and shells ASME Appl. Mech. Rev. 52 305-20

[3] Sunar M and Rao S S 1999 Recent advances in sensing and control of flexible structures via piezoelectric materials technology ASME Appl. Mech. Rev. 52 1-16

[4] Benjeddou A 2002 Advances in piezoelectric finite element modeling of adoptive structural elements: a survey Comput. Struct. 76 347-63

[5] Ray M C, Bhattacharyya R and Samanta B 1994 Static analysis of an intelligent structures by the finite element method Comput. Struct. 52 617-31

[6] Batra R C and Vidoli S 2002 Higher-order piezoelectric plate theory derived from a three-dimensional variational principle AIAA J. 40 91-104

[7] Yang J S 1999 Equations for thick elastic plates with partially electroded piezoelectric actuators and higher order electric fields Smart Mater. Struct. 8 83-91

[8] Bisegna P, Caruso G and Maceri F 2001 A layer-wise Reissner-Mindlin-type model for the vibration analysis and suppression of piezoactuated plates Comput. Struct. 79 2309-19

[9] Mitchell J A and Reddy J N 1995 A refined hybrid plate theory for composite laminates with piezoelectric laminae Int. J. Solids Struct. 32 2345-67

[10] Fukunaga H, Hu N and Ren G X 2001 FEM modeling of adaptive composite structures using a reduced higher-order plate theory via penalty functions Int. J. Solids Struct. 38 8735-52

[11] Xu K, Noor A K and Tang Y Y 1995 Three-dimensional solutions for coupled thermoelectroelastic response of multilayered plates Comput. Methods Appl. Mech. Eng. 126 355-71

[12] Ray M C, Rao K M and Samanta B 1993 Exact solution for static analysis of an intelligent structure under cylindrical bending Comput. Struct. 47 1031-42
[13] Dube G P, Upadhyay M M, Dumir P C and Kumar S 1998 Piezothermoelastic solution for angle-ply laminated plate in cylindrical bending Struct. Eng. Mech. 6 529-42

[14] Vel S S and Batra R C 2000 Cylindrical bending of laminated plates with distributed and segmented piezoelectric actuators/sensors AIAA J. 38 857-67

[15] Fernandes A 2000 Model et etude de composants piezoelectriques: applications aux structures multifonctionnelles PhD Thesis L'Universite Paris VI, France

[16] Raja S, Prathap G and Sinha P K 2002 Active vibration control of composite sandwich beams with piezoelectric extension-bending and shear actuators Smart Mater. Struct. 11 63-71

[17] Lam K Y, Peng X Q, Liu G R and Reddy J N 1997 A finite-element model for piezoelectric composite laminates Smart Mater. Struct. 6 583-91

[18] Trindade M A, Benjeddou A and Ohayon R 2001 Piezoelectric active vibration control of damped sandwich beams J. Sound Vib. 246 653-77

[19] Tzou H S and Tseng C I 1990 Distributed piezoelectric sensor/actuator design for dynamic measurement/control of distributed parameter system: a piezoelectric finite element approach J. Sound Vib. 138 17-34

[20] Koko I S, Orisamolu I R, Smith M J and Alepan U O 1997 Finite element based design tool for smart structures Smart Structures and Materials (Washington) V V Varadan and J Chandra; Proc. SPIE 3039 125-34

[21] Park C, Walz C and Chopra I 1996 Bending and torsion models of beams with induced-strain actuators Smart Mater. Struct. 5 98-113

[22] Spearritt D J and Asokanthan S F 1996 Torsional vibration control of a flexible beam using laminated PVDF actuators J. Sound Vib. 193 941-56

[23] Zhu M L, Lee S W R, Li H L, Zhang T Y and Tong P 2002 Modelling of torsional vibration induced by extension-twisting coupling of anisotropic composite laminates with piezoelectric actuators Smart Mater. Struct. $1155-62$

[24] Ganapathi M, Patel B P, Polit O and Touratier M 1999 A C ${ }^{1}$ finite element including transverse shear and torsion warping for rectangular sandwich beams Int. J. Numer. Methods Eng. 45 47-75

[25] Beakou A and Touratier M 1993 A rectangular finite element for analysing composite multilayered shallow shells in statics, vibration and buckling Int. J. Numer. Methods Eng. 36 627-53

[26] Cheng S, Wei X and Jiang T 1989 Stress distribution and deformation of adhesive-bonded laminated composite beams ASCE J. Eng. Mech. 115 1150-62

[27] Jones R M 1975 Mechanics of Composite Materials (New York: McGraw-Hill)

[28] Wetherhold R C and Panthalingal N 1994 Piezoelectric PZT/epoxy composites for controlling torsional motion J. Intell. Mater. Syst. Struct. 5 576-80 